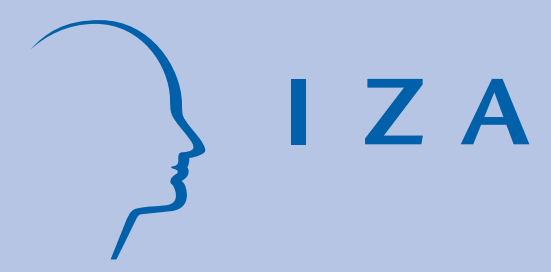

IZA Standpunkte Nr. 11

Grundeinkommen vs. Kombilohn:

Beschäftigungs- und Finanzierungswirkungen und Unterschiede im Empfängerkreis

Clemens Fuest Andreas Peichl

Mai 2009 


\title{
Grundeinkommen vs. Kombilohn: Beschäftigungs- und Finanzierungswirkungen und Unterschiede im Empfängerkreis
}

\author{
Clemens Fuest \\ Oxford University \\ und IZA
}

Andreas Peichl

IZA und ISER

IZA Standpunkte Nr. 11

Mai 2009

IZA

Postfach 7240

53072 Bonn

Tel.: (0228) 3894-0

Fax: (0228) 3894-180

E-Mail: iza@iza.org

Die Schriftenreihe "IZA Standpunkte" veröffentlicht politikrelevante Forschungsarbeiten und Diskussionsbeiträge von IZA-Wissenschaftlern, IZA Research Fellows und IZA Research Affiliates in deutscher Sprache. Die Autoren sind für den Inhalt der publizierten Arbeiten verantwortlich. Im Interesse einer einheitlichen Textzirkulation werden Aktualisierungen einmal publizierter Arbeiten nicht an dieser Stelle vorgenommen, sondern sind gegebenenfalls nur über die Autoren selbst erhältlich. 


\section{ZUSAMMENFASSUNG}

\section{Grundeinkommen vs. Kombilohn: Beschäftigungs- und Finanzierungswirkungen und Unterschiede im Empfängerkreis}

In der ökonomischen Literatur wird oft argumentiert, dass Kombilohnmodelle und Grundeinkommenskonzepte im Prinzip nur Varianten einer negativen Einkommenssteuer seien. Dabei wird oft übersehen, dass diese Konzepte sehr unterschiedliche Gruppen der Bevölkerung einbeziehen. Dieser Beitrag bietet einen empirischen Vergleich der Bezugsgruppen dieser Konzepte. Wir zeigen, dass der Übergang zu einem selbst restriktiv ausgestalteten Grundeinkommen die Zahl der Transferempfänger gegenüber dem Status quo ungefähr verdoppeln würde. Beim Kombilohn würde die Zahl der Transferempfänger hingegen nur um rund zehn Prozent ansteigen. Das hat Folgen für die Finanzierbarkeit und die Beschäftigungswirkungen. Das zentrale Resultat unserer Analyse lautet, dass der Unterschied im Empfängerkreis letztlich die Ursache dafür darstellt, dass Kombilohnkonzepte finanzierbar sind und positive Beschäftigungswirkungen entfalten können, während Grundeinkommenskonzepte entweder nicht finanzierbar sind, oder, wegen der erforderlichen Gegenfinanzierung, negative Beschäftigungswirkungen entfalten.

JEL-Codes: D31, H24, I38

Schlagworte: Grundeinkommen, Bürgergeld, Kombilohn, Empfängerkreis

Kontaktadresse:

Andreas Peichl

IZA

P.O. Box 7240

D-53072 Bonn

E-mail: peichl@iza.org

\footnotetext{
* Wir danken Ingmar Kumpmann, Karlhans Sauernheimer, Thilo Schaefer und Hilmar Schneider für hilfreiche Kommentare und Anregungen.
} 


\section{Einleitung}

Die Bekämpfung der Arbeitslosigkeit gehört zu den wichtigsten wirtschaftspolitischen Herausforderungen. Von dieser Arbeitslosigkeit sind gering qualifizierte Arbeitnehmer in besonderer Weise betroffen. Unzulänglichkeiten des komplexen Steuer- und Transfersystems werden hierbei neben Rigiditäten des Arbeitsmarktes als Hauptursachen für die hohe Arbeitslosigkeit diagnostiziert. Die ökonomischen Konsequenzen eines Transfersystems mit umfangreicher Grundsicherung sind niedrige Arbeitsangebotsanreize und Arbeitslosigkeit für Niedriglohnbezieher bzw. gering qualifizierte Arbeitnehmer. Die hohe Transferentzugsrate und der geringe Lohnabstand verzerren das Arbeitsangebot, da Unternehmen produktivitätsorientierte Löhne zahlen. Als Konsequenz gibt es keine Arbeitsplätze für Personen, deren Lohn bzw. Produktivität geringer als das Grundsicherungsniveau (zuzüglich Arbeitsleid) ist.

Um die Arbeitslosigkeit im Niedriglohnsegment zu bekämpfen, werden derzeit verschiedene Reformen des Steuer- und Transfersystems diskutiert. Das zentrale Problem besteht darin, die Garantie des soziokulturellen Existenzminimums mit hinreichenden Arbeitsangebotsanreizen im Niedriglohnsektor zu kombinieren. Je nach Ausgestaltung kann man verschiedene Konzeptideen unterscheiden: „bedarfsorientierte Grundsicherung" (dies entspricht dem Status quo), „Kombilohn“, und „Grundeinkommen“ (vgl. hierzu z.B. Mitschke (2000) oder Wissenschaftlicher Beirat beim Bundesministerium der Finanzen (2008)). Allen vorgeschlagenen Grundsicherungsreformkonzepten ist gemein, dass sie die beiden Ziele "Garantie eines Mindesteinkommens" und "Verbesserung der Arbeitsanreize im Niedriglohnbereich" verfolgen, wenn auch jeweils mit unterschiedlicher Gewichtung.

Ausgangspunkt der Kombilohndiskussion ist die Diagnose, dass niedrig qualifizierte Arbeitnehmer, wenn sie überhaupt Arbeitsplätze finden, in vielen Fällen Einkommen erzielen, die nicht existenzsichernd sind oder Transfers an Arbeitslose kaum übersteigen. Daher ist der Anreiz, überhaupt nach Arbeit zu suchen, gering. Außerdem sind die Tariflöhne in vielen Branchen in den unteren Lohngruppen so hoch, dass kaum niedrig qualifizierte Arbeitnehmer eingestellt werden. Kombilohnvorschläge verfolgen das Ziel, gering qualifizierten Arbeitnehmern den Arbeitsplatz zu erhalten und denjenigen, die bereits arbeitslos sind oder am Anfang ihres Berufslebens keine Arbeit finden, zu einem Arbeitsplatz im ersten, regulären Arbeitsmarkt zu verhelfen. Das geschieht im Wesentlichen, indem die Arbeitseinkommen durch ergänzende Transfers aufgestockt werden. Dadurch steigen die Anreize, Arbeit auch bei niedrigen Löhnen aufzunehmen.

Darüber hinaus werden immer wieder auch weiterreichende Reformen des Sozialsystems durch die Einführung eines sog. „Grundeinkommens" propagiert. Ein solches Grundeinkommen oder "Bürgergeld“, das allen Bürgern unabhängig von ihren sonstigen persönlichen und wirtschaftlichen Verhältnissen und ohne Gegenleistung ausgezahlt wird, ersetzt die wichtigsten Transferleistungen des Sozialsystems.

In der ökonomischen Literatur wird oft argumentiert, dass Kombilohnmodelle und Grundeinkommenskonzepte im Prinzip nur Varianten einer negativen Einkommenssteuer seien (vgl. hierzu z.B. Bonin und Schneider (2007) oder Erlei (2007)). Das ist zwar insofern richtig, als zum einen ein gewisses Transferniveau für Menschen, die keinerlei eigenes Einkommen erwirtschaften, und zum anderen eine Transferentzugsrate von üblicherweise deutlich unter 100 Prozent beide Konzepte charakterisieren. Hinzu kommt, dass in beiden Konzepten üblicherweise auf eine Bedürftigkeitsprüfung verzichtet wird. ${ }^{1}$

Trotz dieser Gemeinsamkeiten unterscheiden sich die Wirkungen von Kombilohnkonzepten und Grundeinkommenskonzepten sehr stark voneinander. Das liegt daran, dass sie sehr unterschiedliche Teile der Bevölkerung einbeziehen. Beim Kombilohn werden Transfers lediglich an Erwerbstätige gezahlt, während jedermann Anspruch auf das Grundeinkommen hat. Eine systematische Analyse des Empfängerkreises der verschiedenen Grundsicherungskonzepte fehlt bisher in der Literatur. Sie steht im Mittel-

\footnotetext{
${ }^{1}$ Beim Kombilohn ist das letztlich keine Prinzipienfrage, sondern eine Frage der administrativen Praktikabilität. Es existieren allerdings auch Bürgergeldkonzepte, die eine Bedürftigkeitsprüfung beinhalten, so etwa das Bürgergeldkonzept der FDP.
} 
punkt dieses Beitrags. Wir vergleichen die Zahl und die sozioökonomische Zusammensetzung des Kreises der Transferempfänger in einem Kombilohnregime mit dem Empfängerkreis unter einem Grundeinkommenskonzept. Dabei betrachten wir bewusst ein sehr restriktives Grundeinkommen, damit wir dessen Empfängerkreis und die fiskalischen Kosten eher unter- als überschätzen. Unsere empirische Analyse zeigt, dass der Übergang zu einem Grundeinkommen die Zahl der Transferempfänger gegenüber dem Status quo ungefähr verdoppeln würde. Beim Kombilohn würde die Zahl der Transferempfänger hingegen nur um rund zehn Prozent ansteigen. Das hat Folgen für die Finanzierbarkeit und die Beschäftigungswirkungen der Konzepte. Das zentrale Resultat unserer Analyse lautet, dass der Unterschied im Empfängerkreis letztlich die Ursache dafür darstellt, dass Kombilohnkonzepte finanzierbar sind und positive Beschäftigungswirkungen entfalten können, während Grundeinkommenskonzepte entweder nicht finanzierbar sind, oder, wegen der erforderlichen Gegenfinanzierung, negative Beschäftigungswirkungen entfalten.

Der weitere Gang der Untersuchung ist wie folgt: in Abschnitt 2 werden die betrachteten Grundsicherungskonzepte vorgestellt und miteinander verglichen. In Abschnitt 3 wird das verwendete Mikrosimulationsmodell FiFoSiM und die Datengrundlage erläutert, bevor in Abschnitt 4 die Unterschiede und Gemeinsamkeiten im Empfängerkreis herausgearbeitet werden. In Abschnitt 5 erfolgt eine Analyse der Beschäftigungs- und Finanzierungswirkungen der Reformkonzepte, bevor im letzten Abschnitt 6 ein zusammenfassendes Fazit gezogen wird.

\section{Beschreibung der Grundsicherungskonzepte}

\subsection{Status quo: Bedarfsorientierte Grundsicherung}

In der Bundesrepublik gibt es aktuell eine Vielzahl von Behörden, die mehr als 150 steuer- und beitragsfinanzierte Sozialleistungen verwalten (vgl. Mitschke (2000)). Durch die Hartz-Gesetze ${ }^{2}$ wurden u.a. Arbeitslosenhilfe und Sozialhilfe zum neuen Arbeitslosengeld II zusammengelegt. Erwerbsfähige Hilfsbedürftige erhalten ALG II (Regelsatz: 345 (331) Euro für den Haushaltsvorstand der Bedarfsgemeinschaft im Westen (Osten)), während nicht erwerbsfähige Hilfsbedürftige das sog. Sozialgeld in gleicher Höhe erhalten. Die Transferentzugsrate für Hinzuverdienste bzw. zur Anrechnung der Einkünfte der Bedarfsgemeinschaft liegt zwischen $80-100 \%$. Zusätzlich werden die Beiträge zur Krankenkasse sowie ein Wohnkostenzuschuss für Kaltmiete und Heizkosten gezahlt. Der durchschnittliche Leistungsumfang beträgt ca. 630 Euro im Monat für einen Single. Falls ein Hilfsbedürftiger ohne wichtigen Grund die Aufnahme einer zumutbaren Arbeit oder eines „Ein-Euro-Jobs“ verweigert, kann die Regelleistung um 30\%, im Extremfall sogar vollständig gekürzt werden.

Für eine Analyse des Empfängerkreises von Grundsicherungskonzepten ist zunächst von Interesse, wer im heutigen System Transferleistungen bezieht. In der Diskussion um ein bedingungsloses Grundeinkommen wird oft implizit davon ausgegangen, dass alle Personen im unteren Einkommensbereich bereits heute staatliche Unterstützungsleistungen erhalten. Dies ist in der Realität jedoch nicht der Fall. Ein Blick in die Daten bzw. die empirische Literatur identifiziert hierfür im Wesentlichen drei Gründe. Zuerst ist die Betrachtung von Bedarfsgemeinschaften im aktuellen Transfersystem zu nennen. Bei der Prüfung der Anspruchsberechtigung für eine Transferzahlung werden nicht nur die eigenen Einkünfte sondern auch die aller anderen Haushaltsmitglieder berücksichtigt und angerechnet. Zweitens werden auch Vermögenswerte berücksichtigt, so dass es durchaus sein kann, dass jemand in einer Periode zwar keine Einkünfte erzielt, aber aufgrund von Vermögenswerten durchaus in der Lage ist, seinen Lebensunterhalt

\footnotetext{
2 Im Zuge der Hartz-Reformen wurden bis 2003 vier Gesetze umgesetzt. Das so genannte Hartz I-Gesetz befasst sich im Kern mit der Gründung der Personal Service Agenturen zur Neuregelung von Leiharbeit. Mini- und Midijobregelungen sind Hauptbestandteil von Hartz II, dagegen wurde in Hartz III der Schwerpunkt im Umbau der Arbeitsagenturen gesetzt. Im vielfach diskutierten Hartz IV-Gesetz findet sich die Zusammenlegung von Sozialhilfe und Arbeitslosenhilfe wieder. Ebenso sind dort verschärfte Zumutbarkeitskriterien, Vermögensanrechnungen und Zuverdienstgrenzen geregelt (vgl. z.B. Wunderlich (2004)).
} 
zu finanzieren („Entsparen“). Drittens ist auch immer wieder der bewusste oder unbewusste Verzicht eigentlich empfangsberechtigter Personen auf die ihnen zustehenden Transferleistungen zu beobachten. ${ }^{3}$ Mögliche Gründe für einen bewussten Verzicht sind z.B. die Angst vor einem Verlust an sozialem Prestige oder eine relative kurze Dauer der Bedürftigkeit z.B. in Folge eines Arbeitsplatzwechsels. Der unbewusste Verzicht lässt sich durch die Unkenntnis der gesetzlichen Regelungen erklären. In einem Grundeinkommenskonzept, das jedem Bürger unabhängig von dessen sozio-ökonomischen Kontext (Familie, Vermögen) einen monatlichen Transfer gewährt, sind diese Gründe für den Nichtbezug des „Bürgergeldes" nicht mehr gegeben. Dies hat einen stark gestiegenen Empfängerkreis zur Folge.

\subsection{Kombilohn und negative Einkommensteuer}

\subsubsection{Grundidee}

Bisher wurden Kombilohnmodelle in Deutschland (Mainzer Modell, Saar-Modell, Elemente von Hartz IV, so etwa das Einstiegsgeld nach § 29 SGB II, geplante Kombilohn-Modelle des Bundesarbeitsministers, der Länder Nordrhein-Westfalen und Hessen sowie der CDU/CSU) ${ }^{4}$ nur zeitlich und geografisch beschränkt auf einen eng begrenzten Empfängerkreis angewendet. Aktuell existieren Kombilohnmodelle z.B. in den USA (Earned Income Tax Credit), Großbritannien (Working Families' Tax Credit), Belgien, Finnland, Irland, Neuseeland und der Schweiz (vgl. hierzu z.B. Kaltenborn und Knerr (2006)).

Kombilohnmodelle ${ }^{5}$ sind i.d.R. beschäftigungspolitisch motivierte, bedarfsabhängige Lohnsubventionen und basieren auf der Diagnose, dass niedrig qualifizierte Arbeitnehmer entweder überhaupt keine Arbeitsplätze finden ${ }^{6}$ oder aber in vielen Fällen Einkommen erzielen, die nicht existenzsichernd sind oder Transfers an Arbeitslose kaum übersteigen. Die Folge sind geringe Arbeitsanreize für niedrig qualifizierte Personen. Darüber hinaus sind die Tariflöhne im unteren Lohnsegment in vielen Branchen so hoch, dass sie die Produktivität vieler niedrig qualifizierter Arbeitnehmer übersteigen. Hieraus resultiert eine (zu den gegebenen Tariflöhnen) zu geringe Arbeitsnachfrage mit der Folge, dass kaum niedrig qualifizierte Arbeitnehmer eingestellt werden. Mithilfe von Kombilohnmodellen sollen diese Probleme des Arbeitsmarktes behoben werden. Eine Lohnsubvention soll es zum einen gering qualifizierten Arbeitnehmern ermöglichen, ihren Arbeitsplatz zu erhalten (Prävention) und zum anderen denjenigen, die bereits arbeitslos sind oder am Anfang ihres Berufslebens keine Arbeit finden, zu einem Arbeitsplatz im ersten, regulären Arbeitsmarkt verhelfen. Hierfür werden im Wesentlichen den Arbeitnehmern die produktivitätsorientierten Arbeitseinkommen durch ergänzende Transfers aufgestockt. Dadurch steigen die Anreize, Arbeit auch bei niedrigen Löhnen aufzunehmen.

Die grundlegende Idee des Kombilohns, Arbeit statt Arbeitslosigkeit zu finanzieren, findet sowohl in der Wissenschaft als auch in der Politik und der Öffentlichkeit viel Unterstützung. Es gibt aber auch Kritik. Erstens ist nicht zu vermeiden, dass es zu Mitnahmeeffekten kommt, dass also Beschäftigte den Kombilohn in Anspruch nehmen, obwohl sie auch ohne Kombilohn nicht arbeitslos wären. Zweitens besteht die Möglichkeit, dass Arbeitnehmer, die heute ohne Kombilohn einer Vollzeitbeschäftigung nachgehen, es nach Einführung des Kombilohns attraktiver finden, zu einer Teilzeitbeschäftigung überzugehen. Denn

\footnotetext{
${ }^{3}$ Vgl. z.B. Currie (2004) oder Frick und Groh-Samberg (2007), die für Deutschland zeigen, dass im Jahr 2002 bis zu ca. $67 \%$ der Anspruchsberechtigten keine Sozialhilfe beantragen.

${ }^{4}$ Vgl. Gerster und Deubel (1999) (Mainzer Modell); Saar-Gemeinschaftsinitiative (1999); Bundesministerium für Arbeit und Soziales (2006); Ministerium für Arbeit und Gesundheit und Soziales des Landes Nordrhein-Westfalen (2006); Hessische Landesregierung (2006); CDU/CSU (2006).

5 Vgl. Ifo Schnelldienst (2007) für eine aktuelle Übersicht über die verschiedenen Vorschläge.

${ }^{6}$ Der Bundesanteil von Arbeitslosen ohne Berufsausbildung an der Gesamtzahl der Arbeitslosen betrug 2003 34,4\% und stieg im Jahr 2005 auf 40,3\% (jeweils Septemberstand, vgl. www.pub.arbeitsamt.de/hst/services/anba). Der entsprechende Nettozugang an Arbeitslosen ohne Berufsausbildung (Zugänge abzüglich Wiedereingliederungen) betrug in dieser Zeitspanne rund 430000 Arbeitnehmer, jahres-durchschnittlich also etwa 215000 . Der Anteil der Niedriglohnbezieher an den sozialversicherungspflichtig Beschäftigten betrug 2005 22\% (vgl. Bosch und Weinkopf (2007))
} 
die Transfers im Rahmen des Kombilohns reduzieren den damit verbundenen Einkommensverlust. Dem kann man entgegenwirken, indem der Kombilohn nur gezahlt wird, wenn die Empfänger aus eigener Kraft ein gewisses Mindesteinkommen erzielen. Ganz ausschließen lassen sich derartige Reaktionen jedoch nicht.

Kombilohn-Vorschläge werden oft mit einer Arbeitspflicht für arbeitsfähige Transferempfänger kombiniert. Solche Workfare-Konzepte steigern den Anreiz, gering bezahlte Arbeit anzunehmen, indem Leistungen der Grundsicherung an erwerbsfähige Personen nur noch dann gewährt werden, wenn eine Gegenleistung durch Arbeit erbracht wird (vgl. hierzu auch Bonin und Schneider (2006)). Hierzu unterbreitet der Staat jedem, der eine Transferleistung beantragt, ein Beschäftigungsangebot, das in Kombination aus Lohn- und Transferzahlungen das soziale Existenzminimum sicherstellt. Die gegenleistungsfreie Grundsicherung wird somit ersetzt durch eine Grundsicherung für die eine Gegenleistung in Form einer Vollzeittätigkeit zu erfolgen hat.

Durch diese Pflicht zur Annahme von Stellenangeboten und zur Ausübung von Workfare-Tätigkeiten werden auch Arbeitsplätze im Niedriglohnbereich wieder attraktiv, die bei gleichem Zeiteinsatz besser entlohnt werden. Diese Verbesserung der Erwerbsanreize erfolgt selbst dann, wenn das Niveau der Grundsicherung unangetastet bleibt.

\subsubsection{Simulierte Kombilohn-Varianten}

Als Beispiel für einen Kombilohn betrachten wir im Folgenden das Kölner Kombilohnkonzept. ${ }^{7}$ Geringentlohnte, beschäftigte und ungekündigte Arbeitnehmer sowie in eine geringentlohnte Beschäftigung für mindestens ein halbes Jahr kommende Arbeitnehmer haben Anspruch auf eine steuerliche Lohngutschrift. Als gering entlohnt gilt für eine Einzelperson ein Beschäftigungsverhältnis bis zu einem Bruttolohn von 1.400,- $€$ oder einem wöchentlichen Bruttolohn von 350,- $€$. Das gesamte Jahreseinkommen der anspruchserhebenden Einzelperson oder Bedarfsgemeinschaft darf das Doppelte der vorhergesehenen Grundbedarfsbeträge einschließlich der Zuschläge für außergewöhnliche Belastungen nicht überschreiten. Nicht getrennt lebende Ehegatten oder alleinerziehende Elternteile und ihre zum Haushalt gehörenden minderjährigen, unverheirateten Kinder bilden eine Bedarfsgemeinschaft.

Der monatliche Grundbedarf wird einschließlich von pauschalierten Kosten der Warmmiete oder Warmnutzungskosten von Wohnungseigentum festgelegt auf 700,- $€$ für den Haushaltsvorstand, 500,- $€$ für den anderen nicht getrennt lebenden Ehegatten, sowie 300,- $€$ für jedes zur Bedarfsgemeinschaft gehörende Kind. Die Beträge für den Grundbedarf erhöhen sich in Fällen außergewöhnlicher Belastung. Der Gesamtanspruch ist auf $20.000 €$ pro Jahr begrenzt. Die Lohneinkünfte der Einzelperson oder der Bedarfsgemeinschaft werden mit $50 \%$ des Bruttolohns auf die Grundbedarfsbeträge und die Zuschläge für außergewöhnliche Belastung angerechnet (Transferentzugsrate). Der gleiche Satz gilt für den Gesamtbetrag anderweitiger Einkünfte. Hierzu zählen neben sämtlichen anderen Einkunftsarten des Einkommensteuerrechts insbesondere auch anderweitig empfangene Transferzahlungen. Für nicht abhängig Beschäftigte bleiben die bisherigen Sozialtransfersysteme (ALG I, II, Wohngeld) bestehen.

Neben der Basisvariante des Kölner Kombilohnmodells wird auch eine Variante mit der Verknüpfung der gemeinnützigen Arbeit (Workfare-Element) simuliert. Wer keinen Kombilohn erhält, weil er nicht arbeitet obwohl er arbeitsfähig ist, erhält weiterhin die bisherigen Transferleistungen (ALG I, II, Sozialhil-

\footnotetext{
7 Vgl. Fuest et al. (2007a) und Fuest et al. (2007c). Hier sind auch ausführliche Berechnungen der Auswirkungen sowie weitere Varianten zu finden. Die Ergebnisse dort unterscheiden sich jedoch teilweise von den hier berechneten Varianten, zum einem aufgrund einer anderen Arbeitzeitkategorisierung im Arbeitsangebotsmodell und zum anderen aufgrund des hier neu eingeführten Mindesteinkommens. In Fuest et al. (2007a), wurde eine Mindeststundenanzahl von 10 Stunden für Frauen und 25 Stunden für Männer betrachtet. Da Stunden jedoch schwerer als Einkommen zu beobachten sind, wird hier folgende Mindesteinkommens-Regelung betrachtet: Die volle (halbe) Lohngutschrift erhält ein Single-Haushalt bei einem monatlichen Mindesteinkommen von 360 (180) Euro. Für Alleinerziehende beträgt das Mindesteinkommen 240 (120) Euro, bei Paaren ohne Kinder müssen beide Ehegatten die Voraussetzungen eines Singles erfüllen, bei Paaren mit Kindern jeweils einmal Single, einmal Alleinerziehender. Aus diesem Grund weichen die quantitativen Resultate teilweise voneinander ab, die qualitativen Aussagen bleiben jedoch gleich.
} 
fe, Wohngeld). Hierfür ist (von erwerbsfähigen Personen) eine Gegenleistung in Form von gemeinnütziger Arbeit (35 Stunden pro Woche) zu erbringen. Wird diese Gegenleistung nicht erbracht, so reduzieren sich die ausgezahlten Transferleistungen, wie bereits nach geltendem Recht unter bestimmten Bedingungen möglich, auf $70 \%$ des ursprünglichen Niveaus.

\subsection{Bedingungsloses Grundeinkommen}

\subsubsection{Grundidee}

Ein bedingungsloses Grundeinkommen oder „Bürgergeld“, das allen Bürgern unabhängig von ihren sonstigen persönlichen und wirtschaftlichen Verhältnissen und ohne Gegenleistung ausgezahlt wird, ersetzt die wichtigsten Transferleistungen des Sozialsystems und soll die umfassende und lückenlose Sicherung des wirtschaftlichen Existenzminimums aller Bürger gewährleisten. Hierdurch soll es möglich werden, gesellschaftlich wünschenswerte, soziale oder ehrenamtliche Tätigkeiten auszuüben oder Ausund Weiterbildungsphasen abzusichern, ohne ökonomischen Zwängen ausgeliefert zu sein. ${ }^{8}$

Die Grundidee dieser Konzepte geht auf Lady Rhys-Williams (1942) sowie die Ideen zur negativen Einkommensteuer von Friedman (1962) und Tobin (1965) zurück. In Deutschland war Mitschke (1985) einer der ersten Vertreter dieses Konzeptes. Im Zuge dieser Debatte wurden zu Beginn der 1970er Jahre in den USA Experimente zur Einführung eines Grundeinkommens durchgeführt (vgl. z.B. Burtless und Greenberg (1983)). Hierbei zeigten sich deutlich negative Arbeitsangebots- und Anreizwirkungen, so dass von diesen Konzepten Abstand genommen und mit dem Earned Income Tax Credit (EITC) bereits 1973 eine Kombilohnvariante eingeführt wurde. Aktuell existieren solche Grundeinkommenssysteme in Brasilien und Namibia sowie dem US-Bundesstaat Alaska ("Alaska Permanent Fund“). In Alaska wird dieses Grundeinkommen (1100 \$ pro Jahr in 2006) aus den reichhaltigen Rohstoffvorkommen finanziert.

In den letzten Jahren ist die Zahl der Befürworter eines bedingungslosen Grundeinkommens auch in Deutschland stark gestiegen. Die vorgeschlagenen Konzepte unterscheiden sich nicht nur in ihren Ausgestaltungsformen, sondern insbesondere auch in ihrer Rechtfertigung. Neben ideologischen Aspekten wie etwa einer grundlegenden Skepsis gegenüber der Stellung der Erwerbsarbeit in marktwirtschaftlichen Systemen wird insbesondere angeführt, dass mit dem Bürgergeld kein Bürger mehr durch das soziale Netz fallen könnte. Aus ökonomischer Sicht sprechen insbesondere die Entkopplung der Sozialsysteme vom Faktor Arbeit (mit der Folge einer möglichen Senkung der Lohnnebenkosten) sowie eine eventuell mögliche Reduktion der Bürokratie für diese Konzepte. Kritiker verweisen vor allem auf die Frage der Finanzierbarkeit derartiger Konzepte und die problematischen Auswirkungen auf die Arbeitsanreize.

\subsubsection{Die simulierte Bürgergeld-Variante}

Im Folgenden werden die Ausgestaltungsmerkmale des simulierten Bürgergeld-Konzepts kurz erläutert. ${ }^{9}$ Jeder Bürger hat nach seinem 18. Lebensjahr Anspruch auf ein Bürgergeld in Höhe von monatlich $700 €$, das nach Abzug einer Gesundheits- und Pflegeprämie in Höhe von maximal $200 €$ monatlich das soziokulturelle Existenzminimum abdeckt. Für Kinder bis zum vollendeten 18. Lebensjahr erhalten die Eltern ein Bürgergeld von $500 €$. Auch in diesem Betrag ist eine Gesundheitsprämie von $200 €$ enthalten.

\footnotetext{
8 Vgl. Vanderborght und Van Parijs (2005), Blaschke (2005) oder Kumpmann (2006) für einen Überblick über verschiedene Grundeinkommensvorschläge und die verschiedenen Pro- und Contra-Positionen.

${ }^{9}$ Die Modellierung erfolgt in Anlehnung an das Konzept von Dieter Althaus (vgl. hierzu auch Bonin und Schneider (2007), Straubhaar et al. (2007), Opielka und Strengmann-Kuhn (2007), Sachverständigenrat zur Begutachtung der gesamtwirtschaftlichen Entwicklung (2007), S.223ff., und Fuest et al. (2007b)), ohne dieses jedoch exakt widerzuspiegeln. So beträgt das monatliche Bürgergeld im Althaus-Konzept $800 €$ statt $700 €$. Wir wählen $700 €$ um das Bürgergeld zum einen besser mit dem Kombilohn zu vergleichen und es zum anderen (besser) finanzierbar zu machen. Unsere Ergebnisse hier unterscheiden sich von Fuest et al. (2007b) nicht nur durch den niedrigeren Bürgergeldbetrag (und die damit verbundene Verschiebung der Netto-Transferempfangsgrenze von 1.600 auf 1.400 Euro) sondern auch durch die Annahme weiterer Gegenfinanzierung in Form der Lohnsummensteuer der Arbeitgeber.
} 
Der Bürgergeldzahlung wird das erzielte Einkommen gegen gerechnet. Die einheitliche Transferentzugsrate (TER) liegt bei 50 Prozent für Einkommen unter 1.400 Euro brutto im Monat. Für Einkommen über 1.400 Euro brutto im Monat beträgt der einheitliche Grenzsteuersatz 25 Prozent (flat rate tax). Bei Einkünften über 1.400 Euro erhält man das hälftige Bürgergeld als Grundfreibetrag, so dass das Steuersystem (indirekt) progressiv wirkt.

Zur weiteren Finanzierung des Konzeptes unterstellen wir, dass die Arbeitgeber eine Steuer in Höhe ihrer bisherigen Beiträge zu den Sozialversicherungen zahlen. Diese Vorgehensweise hat den Vorteil, dass die Arbeitskosten (und somit die Arbeitsnachfrage) unverändert bleiben.

\subsection{Vergleich der Konzepte}

In Tabelle 1 erfolgt ein Vergleich der drei Konzepte hinsichtlich der wichtigsten Ausgestaltungsparameter.

Tab. 1: Vergleich der Konzeptionen

\begin{tabular}{|l|l|l|l|}
\hline & Grundsicherung (GS, 2.1) & Kombilohn (KL, 2.2) & $\begin{array}{l}\text { Grundeinkommen bzw. } \\
\text { Bürgergeld (BG, 2.3) }\end{array}$ \\
\hline Transfersubjekt & Bedarfsgemeinschaft & Bedarfsgemeinschaft & Individuum \\
\hline Empfängerkreis & Hilfsbedürftige & Geringqualifizierte & Alle \\
\hline Leistungshöhe (Single) & $\begin{array}{l}\text { Ca. 660 } € \text { (Regelsatz und } \\
\text { Unterkunft) }\end{array}$ & $700 €$ & $700 €$ \\
\hline Transferentzugsrate & $80-100 \%$ & $50 \%$ & $50 \%$ \\
\hline Bedürftigkeitsprüfung & $\begin{array}{l}\text { Bedarfsprüfung durch } \\
\text { Sozialbehörden }\end{array}$ & $\begin{array}{l}\text { Einkommensprüfung } \\
\text { durch Finanzamt }\end{array}$ & $\begin{array}{l}\text { Einkommensprüfung } \\
\text { durch Finanzamt }\end{array}$ \\
\hline $\begin{array}{l}\text { Arbeitspflicht für ar- } \\
\text { beitsfähige Personen }\end{array}$ & ja & $\begin{array}{l}\text { teilweise (in „Workfa- } \\
\text { re-Option vorgese- } \\
\text { hen) }\end{array}$ & nein \\
\hline
\end{tabular}

Das Nettoeinkommen eines Singlehaushalts bei unterschiedlichen Bruttoeinkommen wird für den Kombilohn und die Bürgergeld-Variante im Vergleich zum Status quo in Abb. 1 dargestellt. Die jeweiligen Nettoeinkommen wurden unter Berücksichtigung der jeweils gültigen Steuer- und TransfersystemRegelungen mit Hilfe des Mikrosimulationsmodells FiFoSiM (vgl. Abschnitt 3) simuliert. Für den Status quo zeigt sich ein nicht-linearer Verlauf mit einer hohen Transferentzugsrate (fast waagrechter Verlauf). Die Sprungstelle bei $11.200 €$ lässt sich durch die Wohngeldregelung erklären. Für den Kombilohn ergibt sich zunächst ein proportionaler Anstieg des Nettoeinkommens bis die halbe Leistungshöhe von $700 €$ im Monat ( $=4200$ pro Jahr) erreicht ist. Ab diesem Betrag wird das Bruttoeinkommen zur Hälfte angerechnet. Bei einem Bruttoeinkommen von $1400 €$ im Monat (16.800€ pro Jahr) endet die Kombilohnzahlung und die Kurve folgt ab hier dem Status quo-Verlauf. Beim Grundeinkommen werden die $500 €$ im Monat (700€ abzüglich $200 €$ Gesundheits- und Pflegeprämie) direkt ausbezahlt und anschließend das Bruttoeinkommen mit einer Transferentzugsrate von 50\% gegen gerechnet. Bei einem Bruttoeinkommen von $1400 €$ im Monat (16.800€ pro Jahr) endet der Netto-Bürgergeldempfang und der Singlehaushalt fängt an, netto Steuern mit einem Grenzsteuersatz von $25 \%$ zu zahlen. 


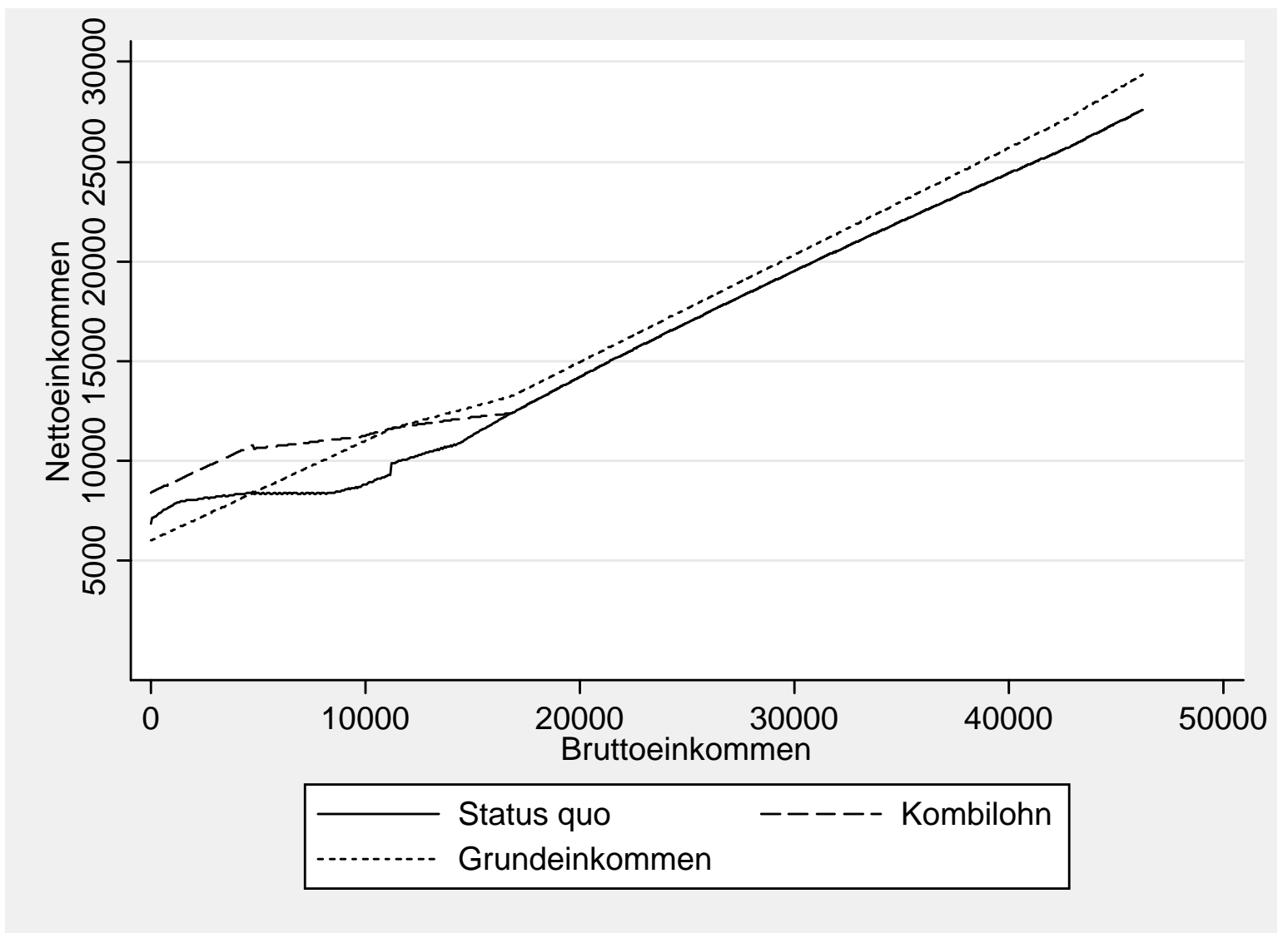

Abb. 1: Darstellung der Konzepte für einen Singlehaushalt

\section{Modellbeschreibung und Datenbasis}

Mittlerweile existieren mehrere Steuer-Transfer-Simulationsmodelle für Deutschland.10 Eine Besonderheit des hier verwendeten Simulationsmodells FiFoSiM ist die Konstruktion einer dualen Datengrundlage unter der Verwendung zweier Mikrodatensätze. Zum einen wird eine 10\%-Stichprobe der Lohn- und Einkommensteuerstatistik von 2001 (FAST01) verwendet und zum anderen das Sozioökonomische Panel (SOEP). Durch die simultane Verwendung beider Datenquellen können fehlende Werte oder Variablen in einer Datenquelle durch Informationen aus der anderen Datenquelle ergänzt werden.

Die Nachbildung des Steuersystems wird mit der Technik der Mikrosimulation vollzogen ${ }^{11}$. Dazu wird im statischen Mikrosimulationsmodul des deutschen Steuer- und Transfersystems von FiFoSiM die indi-

\footnotetext{
${ }^{10}$ Vgl. Peichl (2005) oder Wagenhals (2004), die einen Überblick über Steuer-Transfer-Mikrosimulationsmodelle für Deutschland geben. Diese unterscheiden sich bezüglich der Schätzung von Aufkommenseffekten häufig nur in programmiertechnischen Details, da der Gestaltungsspielraum aufgrund des vorgegebenen gesetzlichen Rahmens begrenzt ist. Größere Unterschiede zwischen den Modellen ergeben sich insbesondere bei der Modellierung von Verhaltensreaktionen.

11 Vgl. Spahn et al. (1992) für eine Einführung in die Technik der Mikrosimulation. Die Simulation einzelner Mikroeinheiten mit ihren individuellen Strukturinformationen ermöglicht eine detaillierte Abbildung der komplexen sozioökonomischen, rechtlichen und institutionellen Zusammenhänge des Steuer- und Transfersystems und somit eine Evaluation persönlich differenzierten Verhaltens.
} 
viduelle Einkommensteuerschuld unter Berücksichtigung von Freibeträgen, Anrechnungspauschalen, Sonderausgaben, sowie Abzugsbeträgen für außergewöhnliche Belastungen und sonstige Privataufwendungen für jeden Fall der Stichprobe berechnet. Anschließend werden die Ergebnisse mit den durch die Fortschreibung angepassten Fallgewichten multipliziert und damit auf die Gesamtpopulation hochgerechnet. Genauso werden für jede betrachtete Reformvariante die individuell zu leistenden Einkommensteuerzahlungen und die Nettoeinkommen der Steuerpflichtigen ermittelt. Auf diese Weise können sowohl die Gesamteffekte als auch die Auswirkungen auf jeden einzelnen Steuerfall analysiert werden.

Um die Effekte auf das Arbeitsangebot zu simulieren, verwenden wir ein diskretes HaushaltsnutzenModell in Anlehnung an Van Soest (1995). Dabei handelt es sich um ein statisches strukturelles Haushaltsarbeitsangebotsmodell, das die Arbeitsangebotsentscheidung der Haushaltsmitglieder als diskretes Problem der Wahl zwischen einer begrenzten Anzahl von möglichen Arbeitszeiten betrachtet. Die Ehepartner treffen ihre Arbeitsangebotsentscheidung gemeinsam; somit können sowohl Wirkungen auf das Arbeitsangebot der Frau als auch auf das des Mannes simuliert werden. Die Verwendung von jeweils 7 diskreten Arbeitszeitkategorien für Männer und Frauen $(0,8,16, \ldots 48)$ führt zu impliziten Mindestarbeitszeiten von 8 Stunden pro Woche.

Das Modell FiFoSiM wird in Fuest et al. (2005) bzw. Peichl und Schaefer (2006) ausführlich dokumentiert.

\section{Verteilungswirkungen und Empfängerkreis}

Der Empfängerkreis eines bedingungslosen Grundeinkommens und eines Kombilohns unterscheidet sich erheblich. Per Definition erhalten das Grundeinkommen zunächst alle Bürger, wobei diejenigen mit höheren Einkommen dies durch Steuerzahlungen zurückzahlen. Dennoch ist auch der Empfängerkreis einer Netto-Bürgergeldzahlung ${ }^{12}$ deutlich größer als im Status quo oder bei einem Kombilohnvorschlag. Im Status quo erhalten rund 9 Mio. Personen eine Transferzahlung (ALG I, II, Sozialhilfe, Wohngeld). Die Lohnsubvention in der Kombilohn-Variante mit gemeinnütziger Arbeit beziehen rund 1,8 Mio. Transfereinheiten, von denen bisher rund 600.000 keine Transferleistungen erhalten haben, so dass die Gesamtzahl an Transferempfängern auf 9,5 Mio. ansteigt. Bürgergeld erhalten dahingegen rund 21,1 Millionen. Ein Großteil dieser zusätzlichen Empfänger macht die Gruppe der Rentner und Pensionäre aus, von denen rund $66 \%$ das Netto-Bürgergeld enthalten. Außerdem erhalten auch Arbeitslose bzw. NichtErwerbstätige diese Transferleistung, während der Kombilohn auf geringentlohnte Beschäftigte beschränkt ist. In Tabelle 2 wird der Empfang der jeweiligen Transferzahlung in Abhängigkeit verschiedener sozio-ökonomischer Merkmale (Haushaltsnettoäquivalenzeinkommen ${ }^{13}$, Alter, Familienstand) präsentiert.

\footnotetext{
12 Hierbei werden nur die Personen berücksichtigt, die tatsächlich (netto) eine Transferzahlung erhalten, nicht jedoch solche Personen, die Netto-Steuerzahler sind und das Bürgergeld als Grundfreibetrag absetzen können.

${ }^{13}$ Wir verwenden die sog. neue OECD-Skala, nach der der Haushaltsvorstand mit einem Gewichtungsfaktor von 1, Haushaltsmitglieder über 14 Jahren mit 0,5, unter 14 mit 0,3 gewichtet werden. Das Nettoeinkommen eines Haushalts wird durch den so ermittelten Äquivalenzfaktor dividiert, um das äquivalenzgewichtete Haushaltseinkommen zu bestimmen.
} 
Tab. 2: Bezug von Transfers

\begin{tabular}{|c|c|c|c|c|}
\hline & $\begin{array}{l}\text { Status quo } \\
(2.1)\end{array}$ & BG (2.3) & $\begin{array}{l}\text { KL+GS14 } \\
(2.2)\end{array}$ & Nur KL \\
\hline gesamt & 18.90 & 44.75 & 20.27 & 3.90 \\
\hline 1 & 32.61 & 99.52 & 33.28 & 2.45 \\
\hline 2 & 72.53 & 99.42 & 73.23 & 13.58 \\
\hline 3 & 28.77 & 86.00 & 33.80 & 10.34 \\
\hline 4 & 14.75 & 52.77 & 16.93 & 5.17 \\
\hline 5 & 8.83 & 29.94 & 10.53 & 3.06 \\
\hline 6 & 10.42 & 36.06 & 11.84 & 2.55 \\
\hline 7 & 5.90 & 31.42 & 7.90 & 2.41 \\
\hline 8 & 5.82 & 10.38 & 5.88 & 0.06 \\
\hline 9 & 6.36 & 1.86 & 6.36 & 0.00 \\
\hline 10 & 5.28 & 0.09 & 5.28 & 0.00 \\
\hline$<25$ & 23.39 & 74.64 & 24.81 & 5.29 \\
\hline $25-45$ & 19.27 & 32.77 & 21.48 & 6.78 \\
\hline $45-65$ & 19.10 & 32.81 & 20.44 & 2.70 \\
\hline$>65$ & 15.56 & 65.89 & 15.56 & 0.00 \\
\hline Single & 18.90 & 47.79 & 20.26 & 4.23 \\
\hline Alleinerziehend & 33.54 & 61.11 & 36.03 & 11.65 \\
\hline Verh. o. Kinder & 14.36 & 43.55 & 14.84 & 0.64 \\
\hline Verh. mit Kindern & 16.88 & 24.62 & 19.14 & 3.15 \\
\hline
\end{tabular}

Tabelle 2 ist wie folgt (zeilenweise) zu interpretieren: Im zweiten Dezil erhalten 72,5\% im Status quo Transferleistungen, im Bürgergeld-Konzept 99,4\%, im Kombilohn-Vorschlag 73,2\%. Zunächst einmal kann man feststellen, dass es in jeder Bevölkerungsgruppe deutlich mehr Bürgergeldbezieher als Kombilohnempfänger oder Transferempfänger im Status quo gibt. Lediglich in den beiden obersten Dezilen, in denen es jedoch keine Kombilohnzahlung mehr gibt, erhalten mehr Haushalte Status-quo-Leistungen als Netto-Bürgergeld. Es zeigt sich, dass sich im Status quo der Transferempfang auf die untersten Dezile konzentriert. Nach Alter und Familienstatus sind die Transferleistungen gleichmäßig verteilt mit Ausnahme der Alleinerziehenden, die deutlich überproportional Unterstützungsleistungen erhalten.

Auf den ersten Blick erscheint der Anteil der Personen, die im untersten Einkommensdezil im Status quo Transferleistungen empfangen als sehr niedrig. Dies lässt sich auf verschiedene Gründe zurückführen. Zum einen spielt die Bedarfgewichtung durch die Bildung von Äquivalenzeinkommen eine Rolle. Doch auch ohne Äquivalenzgewichtung (siehe im Anhang) ist der Anteil der Transferempfänger im untersten Dezil deutlich niedriger als bei einem Grundeinkommen zu erwarten wäre. Ein Blick in die Daten bzw. die empirische Literatur identifiziert hierfür - wie bereits weiter oben erwähnt - im Wesentlichen drei

\footnotetext{
${ }^{14}$ In der Spalte „KL+GS“ sind alle Personen aufgeführt, die unter einem Kombilohn-Regime (KL) Transferleistungen entweder in Form der Lohnsubvention oder aber der bisherigen Transferleistungen (GS = Grundsicherung) erhalten. Die Spalte „Nur KL“
} 
Gründe: die Betrachtung von Bedarfsgemeinschaften, die Anrechnung von Vermögenswerten im aktuellen Transfersystem, sowie der bewusste oder unbewusste Verzicht eigentlich empfangsberechtigter Personen auf die ihnen zustehenden Transferleistungen. Insoweit dieser letzte Effekt nicht zu vernachlässigen ist, ist es im Sinne des Ziels der Mindestsicherung wünschenswert, dass es hier zu einem Anstieg des Transferempfängeranteils kommt, um verdeckte Armut zu bekämpfen. Gleichwohl führt ein Bürgergeld aber auch dazu, dass nicht unbedingt als bedürftig einzustufende Personen mit entsprechenden Vermögenswerten in den Genuss von Nettotransferzahlungen kommen.

Die Personen in den oberen Dezilen, die Bürgergeld erhalten, sind insbesondere Rentenempfänger. Insgesamt zeigt sich, dass ein Grundeinkommenskonzept Transfers an rund $45 \%$ aller Haushalte gewähren würde, während nur 20,3\% der Haushalte im Kombilohnkonzept Transfers (darunter 3,9\% Kombilohnempfänger) erhalten. Da selbst im 8. (äquivalenzgewichteten) Dezil noch rund ein Zehntel der Personen Bürgergeld erhalten, verwundert es kaum, dass sich viele Personen gegen Arbeitsleid und für ein bedingungsloses Grundeinkommen entscheiden. ${ }^{15}$ Die Kombilohnzahlungen konzentrieren sich auf die unteren Einkommensdezile und potentiell einkommensschwache Haushalte (Alleinerziehende). NichtErwerbstätige erhalten per Definition keinen Kombilohn

Wenn man die Verteilung der Transferzahlungen auf unterschiedliche sozio-ökonomische Gruppen betrachtet, fällt auf, dass die größte Gruppe der Bürgergeldempfänger Rentner sind. Beschränkt man die Analyse der Verteilung der Transferempfänger auf erwerbstätige bzw. erwerbsfähige Personen so ergibt sich das in Tabelle 3 präsentierte Bild. Im folgenden Text verstehen wir unter „Erwerbstätigen“ Personen die entweder beschäftigt sind, oder die potentiell dem Arbeitsmarkt zur Verfügung stehen. Hierzu zählen insbesondere nicht Personen in Ausbildung oder Bezieher von Altersrenten. Hierbei ist zu beachten, dass es in den Daten durchaus Personen über 65 Jahre gibt, die dennoch Vollzeit arbeiten. Diese wurden in

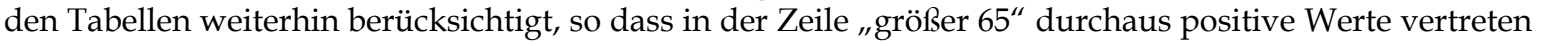
sein können.

15 Hierbei ist zu beachten, dass die bisherigen Darstellungen für die Dezile auf einem äquivalenzgewichteten Nettoeinkommen basierten. Um den Effekt der Äquivalenzgewichtung zu analysieren werden im Anhang die Nettoeinkommensdezile ohne Äquivalenzgewichtung präsentiert (Tabelle 5). Hierbei zeigt sich eine noch stärkere Konzentration der Transferempfänger auf die unteren Dezile in allen Konzepten, die generellen Unterschiede zwischen den Konzepten ändern sich jedoch nicht. 
Tab. 3: Bezug von Transfers (Erwerbsbevölkerung)

\begin{tabular}{|c|c|c|c|c|}
\hline & $\begin{array}{l}\text { Status quo } \\
(2.1)\end{array}$ & BG (2.3) & $\mathrm{KL}+\mathrm{GS}(2.2)$ & Nur KL \\
\hline gesamt & 20.94 & 38.50 & 22.91 & 5.59 \\
\hline 1 & 36.68 & 99.41 & 37.72 & 3.82 \\
\hline 2 & 73.21 & 99.79 & 74.37 & 22.45 \\
\hline 3 & 40.43 & 78.94 & 49.02 & 17.64 \\
\hline 4 & 22.50 & 40.00 & 26.04 & 8.42 \\
\hline 5 & 12.61 & 27.80 & 15.03 & 4.38 \\
\hline 6 & 14.91 & 34.57 & 16.97 & 3.69 \\
\hline 7 & 7.68 & 28.01 & 10.39 & 3.25 \\
\hline 8 & 7.27 & 9.10 & 7.35 & 0.08 \\
\hline 9 & 7.51 & 2.26 & 7.51 & 0.00 \\
\hline 10 & 6.50 & 0.11 & 6.50 & 0.00 \\
\hline$<25$ & 25.61 & 68.30 & 27.59 & 7.37 \\
\hline $25-45$ & 20.37 & 31.92 & 22.90 & 7.76 \\
\hline $45-65$ & 20.52 & 35.39 & 21.98 & 2.96 \\
\hline$>65$ & 15.95 & 65.88 & 15.95 & 0.00 \\
\hline Single & 20.01 & 40.44 & 22.30 & 7.12 \\
\hline Alleinerziehend & 37.66 & 62.94 & 40.72 & 14.28 \\
\hline Verh. o. Kinder & 17.71 & 34.24 & 18.39 & 0.90 \\
\hline Verh. mit Kindern & 17.50 & 25.41 & 19.87 & 3.31 \\
\hline
\end{tabular}

Im Vergleich zu Tabelle 2 ergibt sich in Tabelle 3 (für den zeilenweisen Vergleich einzelner Bevölkerungsgruppen) nur ein geringfügig geändertes Bild, da sich die Population der Rentner über alle Dezile erstreckt. Von den Erwerbspersonen würden insgesamt 38,5\% das Bürgergeld und 22,9\% Transfers im Kombilohnkonzept (darunter 5,6\%, die eine Kombilohnzahlung erhalten) erhalten. Auch für diese Subpopulation gibt es in jeder Bevölkerungsgruppe deutlich mehr Bürgergeldbezieher als Kombilohnempfänger oder Transferempfänger im Status quo.

Es lässt sich somit festhalten, dass es in jeder Bevölkerungsgruppe deutlich mehr Bürgergeldbezieher als Kombilohnempfänger oder Transferempfänger im Status quo gibt. Durch den Übergang zu einem Grundeinkommen würde sich die Zahl der Transferempfänger gegenüber dem Status quo ungefähr verdoppeln, wohingegen bei einem Kombilohn die Zahl der Transferempfänger nur um rund zehn Prozent ansteigen würde. Dies hat Folgen für die Finanzierbarkeit und die Beschäftigungswirkungen der Konzepte, die im folgenden Abschnitt analysiert werden. 


\section{Arbeitsangebots- und Finanzierungswirkungen}

In Tabelle 4 werden die betrachteten Varianten hinsichtlich ihrer Beschäftigungs- ${ }^{16}$ und Kostenwirkungen verglichen.

Tab. 4: Zusammenfassender Vergleich (nach Arbeitsangebotseffekten)

\begin{tabular}{|l||r|r|r|r|}
\hline Modellvariante & $\begin{array}{c}\text { Partizipations- } \\
\text { effekt } \\
\text { in Tsd. }\end{array}$ & $\begin{array}{c}\text { Stundeneffekt } \\
\text { in Tsd. }\end{array}$ & $\begin{array}{c}\text { Gesamter Arbeits- } \\
\text { angebotseffekt } \\
\text { in Tsd. }\end{array}$ & $\begin{array}{c}\text { Nettokosten } \\
\text { in Mrd. }{ }^{17}\end{array}$ \\
\hline \hline Grundeinkommen & 163 & -30 & 133 & 30,3 \\
\hline $\begin{array}{l}\text { Grundeinkommen } \\
\text { Variante (55\% TER) }\end{array}$ & -108 & -167 & -275 & 1,4 \\
\hline \hline Kombilohn & 191 & 12 & 203 & 5,0 \\
\hline KL+Workfare & 524 & 59 & 583 & $-6,8$ \\
\hline
\end{tabular}

Die Werte in der vierten Spalte von Tabelle 4 geben die gesamten Arbeitsangebotsreaktionen umgerechnet in Vollzeitäquivalente an. ${ }^{18}$ Dieser Gesamteffekt setzt sich aus zwei Teileffekten zusammen. Das ist erstens der Partizipationseffekt (zweite Spalte). Hier geht es darum, wie viele Personen (umgerechnet in Vollzeitäquivalente) von Nicht-Erwerbstätigkeit bzw. Arbeitslosigkeit in Erwerbstätigkeit wechseln. Der zweite Teileffekt ist der Stundeneffekt (dritte Spalte). Er beinhaltet die Veränderungen in der Arbeitszeit bisher Beschäftigter (Wechsel von Teilzeit in Vollzeit bzw. umgekehrt). Beide Effekte weisen hier i.d.R. die gleichen Vorzeichen wie der Gesamteffekt auf und der Partizipationseffekt ist jeweils größer. Dies bestätigt die jüngsten Ergebnisse der empirischen Arbeitsangebotsliteratur, nach der das Arbeitsangebot stärker entlang der extensiven als der intensiven Grenze reagiert. ${ }^{19}$

In der Summe führt das Grundeinkommen zu leicht positiven Arbeitsangebotsanreizen. Der Partizipationseffekt ist aufgrund der niedrigeren Transferentzugsrate positiv. Dem gegenüber steht jedoch ein nega-

\footnotetext{
16 Die Berechnungen der Beschäftigungswirkungen in dieser Studie konzentrieren sich auf das Arbeitsangebot. Damit dieses Arbeitsangebot sich tatsächlich in höherer Beschäftigung niederschlägt, müssen auch genug Arbeitsplätze vorhanden sein. Wenn Arbeitslosigkeit auf mangelnde Arbeitsnachfrage in Folge rigider Löhne zurückzuführen ist, können positive Beschäftigungseffekte nur dadurch erreicht werden, dass die Arbeitskosten gesenkt werden und die Unternehmen ihre Nachfrage nach Arbeitskräften ausdehnen.

17 Nettokosten $=$ Neue Transferzahlungen (Kombilohn, Bürgergeld) minus eingesparte Transfers minus Änderung Sozialversicherungsbeiträge minus Änderung Steueraufkommen. Ein negativer Wert signalisiert einen Überschuss.

${ }^{18} \mathrm{Zu}$ beachten ist hierbei, dass durch die im Arbeitsangebotsmodell verwendeten diskreten Arbeitszeitkategorien eine Erwerbstätigkeit von mindestens 8 Wochenstunden angenommen wird.

${ }^{19}$ Vgl. hierzu z.B. Heckman (1993), Blundell und MaCurdy (1999) oder Creedy und Duncan (2002). Stundenelastizitäten werden i.d.R. sowohl für Männer (vgl. Blundell und MaCurdy (1999)) als auch Frauen (vgl. Mroz (1987), Triest (1990)) als nahe Null geschätzt. Im Gegensatz dazu sind die Partizipationselastizitäten stärker ausgeprägt (Heckman (1993)), insbesondere für Personengruppen im unteren Einkommensbereich (vgl. Eissa und Liebman (1996), Meyer und Rosenbaum (2001) oder Immervoll et al. (2007)).
} 
tiver Stundeneffekt, da das hier betrachtete Bürgergeld-Konzept als eine ausgeweitete Grundsicherung konzipiert ist, die den Betroffenen ein höheres Einkommen bei Nicht-Erwerbstätigkeit ermöglicht. In der Folge wird es für einige bisher beschäftigte Personen attraktiver, ihre Stundenzahl zu reduzieren, um in den Genuss des Bürgergeldes zu kommen. ${ }^{20}$

Die Kombilohn-Basisvariante führt zu positiven Beschäftigungswirkungen, wohingegen die Variante mit gemeinnütziger Arbeit zu deutlichen höheren Arbeitsangebotsreaktionen führt. Die Verpflichtung für Empfänger von Arbeitslosenunterstützung, gemeinnützige Arbeit zu leisten (Workfare), erhöht die Bereitschaft, Arbeit im regulären Arbeitsmarkt anzunehmen, deutlich. Es zeigt sich, dass durch großzügigere und umfangreichere Zuschussregelungen auch größere Arbeitsangebotsreaktionen zu erreichen sind (vgl. hierzu auch Fuest et al. (2007c)). Das wirkt sich allerdings auf die Kosten des Kombilohns aus. Deshalb sind neben den Arbeitsangebotsreaktionen insbesondere die fiskalischen Wirkungen von Interesse.

Die Kosten des Grundeinkommens in der fünften Spalte von Tabelle 4 setzen sich zusammen aus dem eigentlichen Bürgergeld, der Gesundheitsprämie und der Rentenzulage, die Einnahmen jeweils aus Einkommensteuer und Arbeitgebersteuer. Zusätzliches Einsparpotential gibt es bei steuerfinanzierten Sozialleistungen, die ganz oder teilweise wegfallen können. ${ }^{21}$ Insgesamt ergibt sich für die Basisvariante eine Finanzierungslücke 30,3 Mrd. Euro nach Arbeitsangebotsreaktionen.

Die Kosten einer Einführung der Kombilohn-Varianten setzen sich zusammen aus den KombilohnZahlungen, abzüglich der eingesparten Transferzahlungen (ALG I, II, Sozialhilfe, Wohngeld) sowie den Änderungen der Einkommensteuer- und Sozialversicherungsbeitragsaufkommen. Das Grundeinkommenskonzept und auch der Kombilohn ohne Workfare führen auch nach Arbeitsangebotsreaktionen zu Nettokosten und relativ hohen Kosten pro Arbeitsplatz. Die Kombilohn-Variante mit Workfare ist deutlich effizienter. Diese positiven Beschäftigungs- und Finanzierungswirkungen haben jedoch den Preis, dass allen Empfängern von Arbeitslosengeld auch eine Arbeitspflicht auferlegt wird. Der damit verbundene bürokratische Aufwand und die möglicherweise bei der Organisation der gemeinnützigen Arbeit anfallenden Kosten sind hier nicht berücksichtigt. Nach unseren Berechnungen würden sich etwa 500.000 Personen für gemeinnützige Arbeit entscheiden. ${ }^{22}$ Das spricht dafür, dass die Organisationskosten erheblich sein könnten. ${ }^{23}$ Diesen Organisationskosten stehen allerdings die Werte gegenüber, die im Rahmen der gemeinnützigen Arbeit geschaffen werden.

In den Fällen, in denen die Vorschläge zu Finanzierungsdefiziten führen, ist jedoch zu berücksichtigen, dass von den Maßnahmen zur Gegenfinanzierung negative Beschäftigungseffekte ausgehen können. Deshalb betrachten wir hier auch eine aufkommensneutrale Grundeinkommensvariante. ${ }^{24}$ Hierbei zeigt sich, dass durch die höhere Grenzbelastung die Anreize zur Arbeitsaufnahme sinken, so dass sich insgesamt negative Arbeitsangebotseffekte i.H.v. 275.000 Vollzeitäquivalenten ergeben.

Insgesamt zeigen unsere Berechnungen, dass der Unterschied im Empfängerkreis letztlich die Ursache dafür darstellt, dass Kombilohnkonzepte, insbesondere wenn sie mit einer Pflicht der arbeitslosen Transferempfänger zu gemeinnütziger Arbeit verbunden werden, finanzierbar sind und positive Beschäfti-

${ }^{20}$ Unsere Analyse des Bürgergeld-Vorschlags von Mitschke (2004) führt zu qualitativ vergleichbaren Arbeitsangebotseffekten (vgl. Bergs et al. (2006)), die in der Summe sogar negativ werden.

${ }^{21}$ Aus Gründen der Vergleichbarkeit unterstellen wir das gleiche Einsparvolumen wie Opielka und StrengmannKuhn (2007) (Tabelle 6, S. 79).

${ }^{22}$ Diese Zahl liegt zwischen den Schätzungen von Bonin und Schneider (2006) (300.000) und Sachverständigenrat zur Begutachtung der gesamtwirtschaftlichen Entwicklung (2005) (700.000).

${ }^{23}$ Laut aktuellen Daten der Bundesagentur für Arbeit gibt es rund 300.000 „1-Euro-Jobs“. Die Nachfrage nach diesen Beschäftigungsmöglichkeiten durch Arbeitslose ist deutlich höher. Weiterhin schätzt die Bundesagentur für Arbeit, dass die Verwaltungs- und Organisationskosten pro 1-Euro-Job rund 350 Euro pro Monat betragen (vgl. hierzu Bundesagentur für Arbeit (2007)). Bei rund 500.000 Workfare-Jobs würden sich somit Kosten in Höhe von rund 2,1 Mrd. Euro pro Jahr ergeben.

${ }^{24}$ Diese aufkommensneutrale Grundeinkommensvariante unterscheidet sich vom bisherigen Konzept durch eine höhere Transferentzugsrate (TER) von 55\% (Grenzsteuersatz damit 27,5\%). 
gungswirkungen entfalten können. ${ }^{25}$ Grundeinkommenskonzepte hingegen sind entweder nicht finanzierbar (bei dann positiven Beschäftigungswirkungen, siehe auch Bonin und Schneider (2007)), oder entfalten - wegen der erforderlichen Gegenfinanzierung- keine bzw. negative Beschäftigungswirkungen. ${ }^{26}$

\section{Fazit}

In der ökonomischen Literatur wird oft argumentiert, dass Kombilohnmodelle und Grundeinkommenskonzepte im Prinzip mit Blick auf die ökonomischen Wirkungen nur Varianten einer negativen Einkommenssteuer sind. Was hierbei jedoch i.d.R. nicht berücksichtigt wird, ist, dass diese Wirkungen insbesondere von den unterschiedlichen Bezugsgruppen der Konzepte bestimmt werden.

Unsere empirische Analyse zeigt, dass der Übergang zu einem Grundeinkommen die Zahl der Transferempfänger gegenüber dem Status quo ungefähr verdoppeln würde. Beim Kombilohn würde die Zahl der Transferempfänger hingegen nur um rund zehn Prozent ansteigen. Für alle betrachteten Bevölkerungsgruppen sind die Anteile der Transferempfänger bei einem bedingungslosen Grundeinkommen am höchsten. Dieser unterschiedliche Empfängerkreis lässt sich insbesondere durch die unterschiedliche Zielgewichtung der Konzepte erklären. Während bei Kombilohnmodellen das Beschäftigungsziel im Vordergrund steht, ist es bei Grundeinkommenskonzepten das Ziel der Mindestsicherung. Dies hat jedoch Folgen für die Finanzierbarkeit und die Beschäftigungswirkungen der Konzepte. Das zentrale Resultat unserer Analyse lautet, dass der Unterschied im Empfängerkreis letztlich die Ursache dafür darstellt, dass Kombilohnkonzepte finanzierbar sind und positive Beschäftigungswirkungen entfalten können, während Grundeinkommenskonzepte entweder nicht finanzierbar sind, oder, wegen der erforderlichen Gegenfinanzierung, negative Beschäftigungswirkungen entfalten.

Andere Untersuchungen belegen vergleichbar ungünstige Auswirkungen ähnlicher Grundeinkommensvorschläge. ${ }^{27}$ Auch die Erfahrungen in anderen Ländern sprechen gegen solche Konzepte. So wurden bereits zu Beginn der 1970er Jahre in den USA Experimente zur Einführung eines Grundeinkommens durchgeführt (vgl. z.B. Burtless und Greenberg (1983)). Hierbei zeigten sich deutlich negative Arbeitsangebots- und Anreizwirkungen, so dass von diesen Konzepten Abstand genommen und mit dem Earned Income Tax Credit (EITC) bereits 1973 eine Kombilohnvariante eingeführt wurde. Bei Kombilöhnen wird eine staatliche Unterstützungsleistung nur dann gewährt, wenn tatsächlich eine Beschäftigung aufgenommen wird. Das hat zwei Vorteile: Erstens werden stärkere Anreize zur Arbeitsaufnahme geschaffen, denn der Einkommensabstand zwischen Erwerbslosigkeit und Arbeitseinkommen vergrößert sich. Zweitens ist der Empfängerkreis deutlich kleiner - Transfers erhält nur, wer arbeitet. Die Folge ist, dass Kombilohnmodelle einfacher finanzierbar sind, da weniger Arbeitslose voll finanziert werden müssen und stattdessen bei Kombilohnempfängern nur eine Teilleistung gezahlt wird. Voraussetzung ist auch bei diesen Ansätzen, dass entsprechende Arbeit zu niedrigen Einkommen nachgefragt wird.

Zusammenfassend lassen sich die unterschiedlichen Wirkungen der Konzepte mit dem unterschiedlichen Empfängerkreis erklären. Per Definition erhalten das Grundeinkommen zunächst alle Bürger, wobei diejenigen mit höheren Einkommen dies durch Steuerzahlungen zurückzahlen, während ein Kombilohn nur für eine ausgewählte Bevölkerungsgruppe in Frage kommt. Die Folge dieses größeren Empfängerkreises sind negative Finanzierungswirkungen und/oder geringere Arbeitsangebotsanreize. Insofern erscheinen Grundeinkommenskonzepte wenig geeignet, um die Massenarbeitslosigkeit gering qualifi-

\footnotetext{
25 Wenn darauf verzichtet wird, sind die Beschäftigungswirkungen geringer und die fiskalischen Risiken erheblich. Dies bestätigt die Schlussfolgerungen des Sachverständigenrates im Zusammenhang mit seinem Kombilohnkonzept, vgl. Sachverständigenrat zur Begutachtung der gesamtwirtschaftlichen Entwicklung (2005), TZ. 540.

${ }^{26}$ Diese Ergebnisse sind robust gegenüber anderen Parameterspezifikationen der beiden Grundeinkommens- bzw. Kombilohnkonzepte. Die qualitativ gleichen Ergebnisse ergeben sich auch für andere, ähnlich ausgestaltete Grundeinkommens- oder Kombilohnmodelle.

27 Vgl. hierzu z.B. Buslei und Steiner (1999), Bergs et al. (2006), Kaltenborn (2000) oder Wissenschaftlicher Beirat beim Bundesministerium der Finanzen (2008).
} 
zierter Personen in Deutschland dauerhaft zu bekämpfen. Kombilohnvorschläge können dies effektiver und effizienter erreichen. Unsere Analyse zeigt allerdings auch, dass bei Kombilohnkonzepten die Beschäftigungswirkungen begrenzt sind, sofern sie nicht mit Workfare kombiniert werden.

\section{Literatur}

Bergs, C., Fuest, C., Heilmann, S., Peichl, A. und Schaefer, T. (2006), Aufkommens-, Beschäftigungs- und Wachstumswirkungen einer Reform des Steuer- und Transfersystems nach dem BürgergeldVorschlag von Joachim Mitschke, FiFo-Bericht 08-2006.

Blaschke, R. (2005), Garantierte Mindesteinkommen. Modelle von Grundsicherungen und Grundeinkommen im Vergleich, Meißen/Dresden.

Blundell, R. und MaCurdy, T. (1999), Labor Supply: A Review of Alternative Approaches, in: Ashenfelter, O. und Card, D. (Hrsg.), Handbook of Labor Economics, Vol. 3A. Elsevier, S. 1559-1695.

Bonin, H. und Schneider, H. (2006), Workfare: Eine wirksame Alternative zum Kombilohn, IZA Discussion Paper No. 2399.

Bonin, H. und Schneider, H. (2007), Beschäftigungswirkungen und fiskalische Effekte einer Einführung des Solidarischen Bürgergelds, www.iza.org.

Bosch, G. und Weinkopf, C. (Eds) (2007), Arbeiten für wenig Geld - Niedriglohnbeschäftigung in Deutschland. Frankfurt/Main, Campus Verlag.

Bundesagentur für Arbeit (2007), Leistungen zur Eingliederung an erwerbsfähige Hilfebedürftige: Einsatz von Arbeitsgelegenheiten 2006, Bericht der Statistik der BA, April 2007.

Bundesministerium für Arbeit und Soziales (2006), Initiative 50 plus: Eckpunkte zur Erhöhung der Beschäftigungsfähigkeit und der Beschäftigungschancen älterer Menschen in Deutschland, Berlin.

Burtless, G. und Greenberg, D. (1983), Measuring the Impact of NIT Experiments on Work Effort. Industrial and Labor Relations Review, Vol. 36, S. 592-605.

Buslei, H. und Steiner, V. (1999), Beschäftigungseffekte von Lohnsubventionen im Niedriglohnbereich, Baden-Baden.

CDU/CSU (2006), CDU und CSU präzisieren Konzept für Geringverdiener. Union plant Lohnzuschüsse von 40 Prozent. Süddeutsche Zeitung, Nr. 107, 10.05.2006, S. 19.

Creedy, J. und Duncan, A. (2002), Behavioural Microsimulation with Labour Supply Responses. Journal of Economic Surveys, Vol. 16(1), S. 1-39.

Currie, J. (2004), The Take Up of Social Benefits, NBER Working Papers 10488. 
Eissa, N. und Liebman, J. B. (1996), Labor supply response to the earned income tax credit. Quarterly Journal of Economics, Vol. 111, S. 605-637.

Erlei, M. (2007), Kombilöhne als Instrument zur Bekämpfung der Arbeitslosigkeit im Niedriglohnbereich, Working paper, TU Clausthal.

Frick, J. R. und Groh-Samberg, O. (2007), To Claim or Not to Claim: Estimating Non-Take-Up of Social Assistance in Germany and the Role of Measurement Error. SOEPpapers on Multidisciplinary Panel Data Research, Vol. 53.

Friedman, M. (1962), Capitalism and Freedom, Chicago

Fuest, C., Mitschke, J., Peichl, A. und Schaefer, T. (2007a), Wider die Arbeitslosigkeit der beruflich Geringqualifizierten: Entwurf eines Kombilohn-Verfahrens für den Niedriglohnsektor. FiFo-CPE Discussion Paper, Vol. 07-01.

Fuest, C., Peichl, A. und Schaefer, T. (2005), Dokumentation FiFoSiM: Integriertes Steuer-TransferMikrosimulations- und CGE-Modell, Finanzwissenschaftliche Diskussionsbeiträge Nr. 05 - 03.

Fuest, C., Peichl, A. und Schaefer, T. (2007b), Beschäftigungs- und Finanzierungswirkungen des Bürgergeld-Konzepts von Dieter Althaus. ifo Schnelldienst, Vol. 60, S. 36-40.

Fuest, C., Peichl, A. und Schaefer, T. (2007c), Beschäftigungs- und Finanzierungswirkungen von Kombilohnmodellen. Wirtschaftsdienst, Vol. 87, S. 226-231.

Gerster, F. und Deubel, I. (1999), Arbeit muss sich lohnen! Das Mainzer Modell für Beschäftigung und Familienförderung. Wirtschaftsdienst, Vol. 79, S. S. 39-49.

Heckman, J. J. (1993), What Has Been Learned About Labor Supply in the Past Twenty Years? The American Economic Review, Vol. 83, S. 116--121.

Hessische Landesregierung (2006), Roland Koch: "Erfahrung hat Zukunft"; Sonderprogramm für ältere Arbeitslose. Newsletter vom 08.09.2006.

Ifo Schnelldienst (2007), Reformkonzepte zur Erhöhung der Beschäftigung im Niedriglohnbereich. Sonderausgabe 4 /.

Immervoll, H., Kleven, H. J., Kreiner, C. T. und Saez, E. (2007), Welfare reform in European countries: a microsimulation analysis. The Economic Journal, Vol. 117, S. 1--44.

Kaltenborn, B. (2000), Reformkonzepte für die Sozialhilfe und ihre Konsequenzen für Fiskus und Arbeitsangebot. Mikroökonometrische Analyse mit dem Sozio-ökonomischen Panel 1986 bis 1996 und dem Simulationsmodell SimTrans. Mitteilungen aus der Arbeitsmarkt- und Berufsforschung, Vol. 33(1), S. 68-79.

Kaltenborn, B. und Knerr, P. (2006), Arbeitsanreize im Niedriglohnbereich - ein ausgewählter internationaler Vergleich, Beiträge zur Wirtschaftsforschung und Politikberatung 30. Berlin. 
Kumpmann, I. (2006), Das Grundeinkommen - Potenziale und Grenzen eines Reformvorschlags. Wirtschaftsdienst, Vol. 86, S. 595-601.

Meyer, B. D. und Rosenbaum, D. T. (2001), Welfare, the earned income tax credit, and the labor supply of single mothers. Quarterly Journal of Economics, Vol. 116, S. 1063-1114.

Ministerium für Arbeit und Gesundheit und Soziales des Landes Nordrhein-Westfalen (2006), Eckpunkte zum Kombilohn. www.gib.nrw.de/specials/kombilohn.

Mitschke, J. (1985), Steuer- und Transferordnung aus einem Guß: Entwurf einer Neugestaltung der direkten Steuern und Sozialtransfers in der Bundesrepublik Deutschland, Baden-Baden.

Mitschke, J. (2000), Grundsicherungsmodelle - Ziele, Gestaltung, Wirkungen, Finanzbedarf. Eine Fundamentalanalyse mit besonderem Bezug auf die Steuer- und Sozialordnung sowie den Arbeitsmarkt der Republik Österreich,

Mitschke, J. (2004), Erneuerung des deutschen Einkommensteuerrechts: Gesetzestextentwurf und Begründung, Köln.

Mroz, T. A. (1987), The sensitivity of an empirical model of married women's hours of work to economic and statistical assumptions. Econometrica, Vol. 55, S. 765-799.

Opielka, M. und Strengmann-Kuhn, W. (2007), Das Solidarische Bürgergeld - Finanz- und sozialpolitische Analyse eines Reformkonzepts, in: Borchard, M. (Hrsg.), Das Solidarische Bürgergeld, Analysen einer Reformidee. S. 13-141.

Peichl, A. (2005), Die Evaluation von Steuerreformen durch Simulationsmodelle, Finanzwissenschaftliche Diskussionsbeiträge Nr. 05-01, Universität Köln.

Peichl, A. und Schaefer, T. (2006), Documentation FiFoSiM: Integrated tax benefit microsimulation and CGE model. FiFo-CPE Discussion Paper, Vol. 06-10.

Rhys-Williams, J. E. (1942), Something to Look Forward to, London.

Saar-Gemeinschaftsinitiative (1999), Modellversuch zur Schaffung zusätzlicher Arbeitsplätze für Geringqualifizierte, Saarlandes, I. d., Arbeit für Einfachqualifizierte - Modellversuch für mehr Beschäftigung. Saarbrücken

Sachverständigenrat zur Begutachtung der gesamtwirtschaftlichen Entwicklung (2005), Jahresgutachten 2005/2006: Die Chance nutzen - Reformen mutig voranbringen, Stuttgart.

Sachverständigenrat zur Begutachtung der gesamtwirtschaftlichen Entwicklung (2007), Jahresgutachten 2007/2008: Das Erreichte nicht verspielen, Stuttgart.

Spahn, P., Galler, H., Kaiser, H., Kassella, T. und Merz, J. (1992), Mikrosimulation in der Steuerpolitik, Heidelberg.

Straubhaar, T., Hohenleitner, I., Opielka, M. und Schramm, M. (2007), Bedingungsloses Grundeinkommen und solidarisches Bürgergeld - mehr als sozialutopische Konzepte. 
Tobin, J. (1965), On the Economic Status of the Negro. Daedalus, Vol. 94, S. 878-895.

Triest, R. K. (1990), The effect of income taxation on labor supply in the United States. Journal of Human Resources, Vol. 25, S. 491-516.

Van Soest, A. (1995), Structural Models of Family Labor Supply: A Discrete Choice Approach. Journal of Human Resources, Vol. 30, S. 63-88.

Vanderborght, Y. und Van Parijs, P. (2005), Ein Grundeinkommen für alle? Geschichte und Zukunft eines radikalen Vorschlags, Frankfurt / Main.

Wagenhals, G. (2004), Tax-benefit microsimulation models for Germany: A Survey. IAW-Report / Institut fuer Angewandte Wirtschaftsforschung (Tübingen), Vol. 32, S. 55-74.

Wissenschaftlicher Beirat beim Bundesministerium der Finanzen (2008), Existenzsicherung und Erwerbsanreiz, Gutachten des Wissenschaftlichen Beirats beim Bundesministerium der Finanzen, Berlin.

Wunderlich, G. (2004), Die Hartz-Gesetze I-IV, in: Hagen, T. und Spermann, A. (Hrsg.), Hartz-Gesetze Methodische Ansätze zu einer Evaluierung. Baden- Baden, Nomos. 


\section{Anhang: Bezug von Transfers ohne Äquivalenzgewich- tung}

Tab. 5: Bezug von Transfers (ohne Äquivalenzgewichtung)

\begin{tabular}{|r||r|r|r|r|}
\hline & \multicolumn{1}{|l|}{$\begin{array}{l}\text { Status quo (ALG } \\
\text { I, II, WG) }\end{array}$} & BG & \multicolumn{2}{l|}{ KL } \\
\hline \hline gesamt & 18.90 & 44.75 & 20.27 & 3.90 \\
\hline \hline 1 & 48.51 & 99.47 & 49.25 & 9.25 \\
\hline 2 & 34.89 & 84.87 & 38.83 & 5.16 \\
\hline 3 & 12.12 & 43.96 & 14.39 & 2.64 \\
\hline 5 & 10.45 & 33.51 & 11.82 & 2.85 \\
\hline 6 & 7.85 & 34.99 & 10.14 & 1.79 \\
\hline 7 & 6.51 & 32.88 & 7.88 & 0.11 \\
\hline 8 & 5.77 & 15.90 & 5.88 & 0.00 \\
\hline 9 & 6.28 & 3.21 & 6.28 & 0.00 \\
\hline 10 & 6.03 & 1.44 & 6.03 & 0.00 \\
\hline
\end{tabular}

